\title{
Investment timing, capacity sizing, and technology choice of power plants
}

\author{
Ryuta Takashima1,*, Afzal S. Siddiqui ${ }^{2,3}$, Shoji Nakada ${ }^{4}$ \\ ${ }^{1}$ Department of Risk Science in Finance and Management, Chiba Institute of Technology, \\ 2-17-1 Tsudanuma, Narashino-shi, Chiba 275-0016, Japan \\ ${ }^{2}$ Department of Statistical Science, University College London, \\ Gower Street, London WC1E 6BT, UK \\ ${ }^{3}$ Department of Computer and Systems Sciences, Stockholm University/KTH \\ Stockholm, Sweden \\ ${ }^{4}$ Department of Nuclear Engineering and Management, The University of Tokyo, \\ 7-3-1 Hongo, Bunkyo-ku, Tokyo 113-8656, Japan
}

\begin{abstract}
Deregulation of electricity industries has created wholesale markets with uncertain prices and offered greater flexibility to investors to make decisions. In this paper, we consider the problem of a typical investor who has discretion over not only the timing, but also the sizing of a new power plant. The interaction between these two types of managerial flexibility may be addressed analytically using the real options approach. Since an investor may also have discretion over technology choice, we allow for an investment opportunity in two mutually exclusive projects with embedded timing and sizing options. Via numerical examples, we illustrate how an investor may make decisions about timing, sizing, and technology choice. Sensitivity analyses to key parameters also highlight the intuition for how decisions are made.
\end{abstract}

Keywords: Power plants; electricity price; capacity sizing; technology choice; real options

\footnotetext{
* Corresponding author

Address: 2-17-1 Tsudanuma, Narashino-shi, Chiba 275-0016, Japan

Phone: +81-47-478-4753; E-mail: takashima@sun.it-chiba.ac.jp
} 


\section{Introduction}

Reform of electricity industries worldwide has been based on the premise that greater economic efficiency may be achieved if participants were allowed to make investment and operational decisions based on price signals. In particular, while the transmission sector still requires regulatory oversight due to its natural monopoly characteristics, there is no reason for the potentially competitive generation and retail sectors to be subject to state regulation (see Wilson [1]). Hence, even though the outcome of these reforms is subject to debate (see Hyman [2]), there is a greater role for markets now.

In tandem with these reforms, a variety of spot and forward markets for electricity have been set up in order to reflect its relative scarcity. Unlike in the vertically integrated paradigm, generators and retailers must account for wholesale prices when making their investment and operational decisions. These prices may be subject to great uncertainty, which makes the timing of investment and operational decisions and the role of managerial flexibility more crucial. Consequently, traditional methods, such as now-or-never net present value (NPV) and internal rate of return (IRR), may be inadequate to cope with dynamic aspects of existing electricity markets, and decision support based on real options (see Dixit and Pindyck [3] and McDonald and Siegel [4]) is one of the alternatives that has been proposed.

The amenability of the real options approach to the energy sector has been widely demonstrated at least as far back as Ekern [5]. Recent work has explored the valuation of generation and transmission assets (Deng et al. [6]), optimal operation of power plants (Tseng and Barz [7] and Deng and Oren [8]), and incentives for construction of nuclear power plants (Rothwell [9]). Modeling decision-making for investment opportunities with embedded options to abandon, expand, or switch projects after adoption is particularly relevant in the electricity industry. Towards this end, real options has been applied to problems of modularity (Gollier et al. [10], Näsäkkälä and Fleten [11], and Siddiqui and Maribu [12]), capacity sizing (Bøckman et al. [13]), and equipment replacement (Takashima et al. [14]). Furthermore, recent advances in real options theory, which address the selection of mutually exclusive projects (Décamps et al. [15]) and the game-theoretic implications of duopolistic competition (Huisman and Kort [16]), have been applied to problems of technology choice (Fleten et al. [17] and Wickart and Madlener [18]) and strategic investment (Takashima et al. [19]).

Here, we take the perspective of a firm that has the perpetual right, but not the obligation, to invest in a power plant. After the plant is constructed, it will operate continuously for a fixed number of years before being decommissioned. During its operating lifetime, the plant will provide the firm with a profit flow related to the stochastic electricity price. We assume that the plant's operating cost is constant, and its construction cost depends on its size. Thus, the firm's problem is to determine not only when to invest, but also its plant's capacity. Furthermore, it may choose between two types of technologies, e.g., nuclear or natural gas. We illustrate how the real options method is able to provide decision support to handle the three types of managerial flexibility (timing, sizing, and technology choice) given a stochastic electricity price, which would not have been possible via traditional valuation tools.

The structure of this paper is as follows:

- Sect. 2 provides the assumptions for the model 
- Sect. 3 introduces the basic real options model for investment under uncertainty with fixed capacity for a single technology

- Sect. 4 formulates and solves the problem with endogenous capacity sizing

- Sect. 5 extends the model to enable mutually exclusive investment in two types of technologies with discretion over timing and sizing

- Sect. 6 provides numerical examples to illustrate the concepts

- Sect. 7 summarizes the results of this paper

\section{Model Setup}

Consider a firm that starts operating a power plant of production output $Q$ (in MWh/annum) by incurring investment cost $I(Q)$ (in $\$$ ) that is a non-linear function of $Q$, i.e., $I(Q)=\delta Q^{\epsilon}$, where $\delta>0$ and $\epsilon>1$. We assume that the investment cost function is convex in output due to diminishing marginal returns. After the investment decision and the construction lead time, $T$ (in years), instantaneous cash flow, $\pi_{t}$, is generated from the power plant over its lifetime, $L$ (in years).

Suppose that the investment decision and the output capacity are dependent on the dynamics of the electricity price. If we assume that the firm is a price taker, i.e., its actions have no influence on the dynamics of the electricity price, then the electricity price, $P_{t}$ (in $\$ / \mathrm{MWh}$ ), may be considered as exogenous. Here, we model it as evolving according to a geometric Brownian motion (GBM) process,

$$
d P_{t}=\mu P_{t} d t+\sigma P_{t} d W_{t}, \quad P_{0}=p,
$$

where $\mu$ and $\sigma$ are the risk-adjusted expected growth rate and the volatility of $P_{t}$, respectively, and $W_{t}$ is a standard Brownian motion.

Consequently, the profit flow can be represented by the following equation,

$$
\pi_{t} \equiv \pi\left(P_{t}, Q\right)=\left(P_{t}-c\right) Q
$$

where $c$ is the operating cost (in $\$ / \mathrm{MWh}$ ) that is composed of the fuel cost as well as operating and maintenance costs. If the plant has operating flexibility, then the profit flow $\pi\left(P_{t}, Q\right)$ becomes zero when $P_{t}<c$. For simplicity, however, we assume that the power plant does not have operating flexibiltiy.

\section{Basic Model}

We begin by describing the model of Gollier et al. [10] that extends the McDonald and Siegel model [4] deriving the investment timing and its option value by introducing fixed construction time and project lifetime. Suppose that the firm can determine the investment timing of a power plant with a fixed output, $Q$. The value of the investment opportunity is:

$$
F(p) \equiv \sup _{\tau \in \mathcal{S}} \mathbb{E}_{p}\left[\int_{\tau+T}^{\tau+T+L} e^{-r t} \pi\left(P_{t}, Q\right) d t-e^{-r \tau} I(Q)\right]
$$


where $\tau$ is the investment time, $\mathcal{S}$ is the set of stopping times of the filtration generated by the electricity price process, and $r>0$ is the risk-free rate. We must have $r>\mu$ in order to ensure that the firm's value is finite for $L \rightarrow \infty$.

Given the investment threshold, $P^{*}$, the optimal investment time, $\tau^{*}$, has the following form:

$$
\tau^{*}=\inf \left\{t \geq 0 \mid P_{t} \geq P^{*}\right\} .
$$

Prior to determining $P^{*}$ and $F(p)$, we calculate the now-or-never expected NPV, $V(p, Q)$, of a power plant with fixed output, $Q$ :

$$
\begin{aligned}
V(p, Q) & =\mathbb{E}_{p}\left[\int_{T}^{T+L} e^{-r t} \pi\left(P_{t}, Q\right) d t-I(Q)\right] \\
& =K_{1} \frac{Q p}{r-\mu}-K_{2} \frac{c Q}{r}-\delta Q^{\epsilon},
\end{aligned}
$$

where $K_{1}=e^{-(r-\mu) T}\left(1-e^{-(r-\mu) L}\right)$ and $K_{2}=e^{-r T}\left(1-e^{-r L}\right)$. Note that as $T \rightarrow 0$ and $L \rightarrow \infty$, both $K_{1}$ and $K_{2}$ tend to one, i.e., we have the case of an infinitely lived power plant that is constructed with no lead time.

The value of the investment opportunity is then determined using the standard methodology as in Dixit and Pindyck [3]. In particular, this is an optimal stopping time problem, which is cast as a non-linear maximization problem using the conditional expectation of the stochastic discount factor, $\mathbb{E}_{p}\left[e^{-r \tau}\right]$, and applying the strong Markov property of the GBM process along with the law of iterated expectations:

$$
\begin{aligned}
F(p) & =\sup _{\tau \in \mathcal{S}} \mathbb{E}_{p}\left[e^{-r \tau} V\left(P_{\tau}, Q\right)\right] \\
& =\max _{P^{*} \geq p}\left(\frac{p}{P^{*}}\right)^{\beta_{1}} V\left(P^{*}, Q\right),
\end{aligned}
$$

where $\beta_{1}>1$ is the positive root of the characteristic equation $\frac{1}{2} \sigma^{2} \beta(\beta-1)+\mu \beta-r=0$. The optimal investment threshold after taking the first-order necessary condition is:

$$
P^{*}=\frac{\beta_{1}}{\beta_{1}-1} \frac{r-\mu}{K_{1}}\left(\frac{c K_{2}}{r}+\delta Q^{\epsilon-1}\right)
$$

By contrast, the now-or-never investment threshold price is $P^{N N}=\frac{r-\mu}{K_{1}}\left(\frac{c K_{2}}{r}+\delta Q^{\epsilon-1}\right)<P^{*}$, i.e., having the deferral option provides a value to waiting, which then increases the opportunity cost of investing.

\section{Investment Timing and Sizing}

In this section, following Dangl $[20]^{1}$, we develop a model for analyzing not only the investment timing, but also the plant sizing. The value of the investment opportunity is now:

$$
\begin{aligned}
F(p) & \equiv \sup _{\tau \in \mathcal{S}, Q \geq 0} \mathbb{E}_{p}\left[\int_{\tau+T}^{\tau+T+L} e^{-r t} \pi\left(P_{t}, Q\right) d t-e^{-r \tau} I(Q)\right] \\
& =\sup _{\tau \in \mathcal{S}} \mathbb{E}_{p}\left[e^{-r \tau} V\left(P_{\tau}, Q^{*}\left(P_{\tau}\right)\right)\right] \\
& =\max _{P^{*} \geq p}\left(\frac{p}{P^{*}}\right)^{\beta_{1}} V\left(P^{*}, Q^{*}\left(P^{*}\right)\right)
\end{aligned}
$$

\footnotetext{
${ }^{1}$ Although Dangl [20] considers the investment timing and the plant sizing with operational flexibility, as described previously, in this paper we assume that the power plant does not have such discretion.
} 
Here, the firm first optimizes the plant's productive capacity, $Q^{*}(p)$, before deciding on investment timing. Thus, the optimal size of the plant for any $p$ is:

$$
\begin{aligned}
Q^{*}(p) & =\arg \max _{Q \geq 0} K_{1} \frac{Q p}{r-\mu}-K_{2} \frac{c Q}{r}-\delta Q^{\epsilon} \\
& =\left[\frac{1}{\delta \epsilon} \max \left(\frac{K_{1} p}{r-\mu}-\frac{K_{2} c}{r}, 0\right)\right]^{\frac{1}{\epsilon-1}}
\end{aligned}
$$

When we substitute the optimal size of the plant for any $p$, Eq. (9), back into the now-or-never expected NPV, Eq. 5, we obtain the maximized now-or-never expected NPV:

$$
\begin{aligned}
V^{*}(p) & \equiv V\left(p, Q^{*}(p)\right) \\
& =\left(\frac{1}{\delta \epsilon}\right)^{\frac{1}{\epsilon-1}}\left(\frac{\epsilon-1}{\epsilon}\right)\left[\max \left(\frac{K_{1} p}{r-\mu}-\frac{K_{2} c}{r}, 0\right)\right]^{\frac{\epsilon}{\epsilon-1}}
\end{aligned}
$$

Inserting Eq. (10) into Eq. (8), we are now able to solve the investment timing problem with endogenous capacity sizing:

$$
F(p)=\max _{P^{*} \geq p}\left(\frac{p}{P^{*}}\right)^{\beta_{1}} V^{*}\left(P^{*}\right)
$$

Taking the first-order necessary condition as before and solving for $P^{*}$ and $Q^{*} \equiv Q\left(P^{*}\right)$, we obtain the following:

$$
\begin{gathered}
P^{*}=\frac{\beta_{1}(\epsilon-1)}{\beta_{1}(\epsilon-1)-\epsilon}(r-\mu) \frac{K_{2} c}{K_{1} r} \\
Q^{*}=\left(\frac{1}{\delta \epsilon}\right)^{\frac{1}{\epsilon-1}}\left[\frac{K_{2} c \epsilon}{r\left(\beta_{1}(\epsilon-1)-\epsilon\right)}\right]^{\frac{1}{\epsilon-1}}
\end{gathered}
$$

We must have $\beta_{1}(\epsilon-1)-\epsilon>0$ to ensure that that $P^{*}$ and $Q^{*}$ are non-negative. However, if $p>P^{*}$, then it is optimal to invest immediately and to construct a plant of size greater than $Q^{*}$. In particular, Eq. (9) would be used to determine the optimal capacity.

\section{Technology Choice}

We now consider the full investment problem in which the firm also has a choice of two technologies for power plants: the first type is capital intensive, but with low operating costs, while the second one has low capital, but high operating, costs. Thus, the first technology may be thought of as nuclear power, while the second one may be based on natural gas combined-cycle combustion. In order to make the tradeoff relevant, we have $c_{N}<c_{G}$ and $\delta_{N}>\delta_{G}$. Other aspects of both projects remain identical, i.e., both types of power plants face the same price shocks, construction lead times, and operating lifetimes. We follow the framework of [15] in order to analyze this problem of mutually exclusive investment in two projects. Unlike [15], we also have the issue of endogenous capacity sizing in addition to investment timing and technology choice.

Formally, the value of investment opportunity here is:

$$
F(p) \equiv \sup _{\tau \in \mathcal{S}, Q \geq 0} \mathbb{E}_{p}\left[\mathbf{1}_{\left\{\tau_{N} \leq \tau_{G}\right\}} e^{-r \tau_{N}} V_{N}\left(P_{\tau_{N}}, Q_{N}^{*}\left(P_{\tau_{N}}\right)\right)+\mathbf{1}_{\left\{\tau_{N}>\tau_{G}\right\}} e^{-r \tau_{G}} V_{G}\left(P_{\tau_{G}}, Q_{G}^{*}\left(P_{\tau_{G}}\right)\right)\right],
$$


where $\mathbf{1}_{\{\cdot\}}$ is the indicator function, $\tau_{i}, i=N, G$, is the investment time of technology $i$ in the case where the firm has two alternative plants, and $\tau$ is the investment time of a plant for either technology, i.e.,

$$
\tau=\min \left\{\tau_{N}, \tau_{G}\right\} .
$$

In order to solve this problem, we first let $\tilde{P}$ be the indifference price between the two projects, i.e., $V_{N}^{*}(\tilde{P})=V_{G}^{*}(\tilde{P})$. Next, we note that if the nuclear power plant has a higher maximized expected NPV than the one for the natural gas plant for some $p<\tilde{P}$, then the option value for the entire investment opportunity, $F(p)$, may be dichotomous. The procedure for checking it is as follows:

1. If $F_{G}(p)>F_{N}(p)$, then $F(p)=F_{G}(p)$;

2. Else $F(p)=F_{N}(p)$ for $0 \leq p<P_{N}^{*}$ and $F(p)=D_{1} p^{\beta_{1}}+D_{2} p^{\beta_{2}}$ for $P_{L}^{*}<p<P_{R}^{*}$

In the first case, it is optimal to skip the nuclear power plant and focus on the gas one. Consequently, the option value is simply the value of the opportunity to invest in the gas power plant. By contrast, in the second case, it may be optimal to invest in the nuclear power plant for $P_{N}^{*} \leq p \leq P_{L}^{*}$. Thus, the option value is dichotomous: there is a lower waiting region for the opportunity to invest only in the nuclear power plant should the price increase sufficiently as well as an upper waiting region around the indifference price in which it may be optimal to invest in either technology. This latter waiting region for $P_{L}^{*}<p<P_{R}^{*}$ has an option value that reflects the opportunity to invest in either gas or nuclear power plants via $D_{1} p^{\beta_{1}}$ and $D_{2} p^{\beta_{2}}$, respectively, where $\beta_{2}$ is the negative root of $\frac{1}{2} \sigma^{2} \beta(\beta-1)+\mu \beta-r=0$, and $D_{1}$ and $D_{2}$ are both positive endogenous constants.

The investment threshold prices, $P_{L}^{*}$ and $P_{R}^{*}$, along with endogenous constants, $D_{1}$ and $D_{2}$, are determined via the following value-matching and smooth-pasting conditions:

$$
\begin{aligned}
F\left(P_{L}^{*}\right) & =V_{N}^{*}\left(P_{L}^{*}\right) \\
F^{\prime}\left(P_{L}^{*}\right) & =V_{N}^{* *}\left(P_{L}^{*}\right) \\
F\left(P_{R}^{*}\right) & =V_{G}^{*}\left(P_{R}^{*}\right) \\
F^{\prime}\left(P_{R}^{*}\right) & =V_{G}^{*}\left(P_{R}^{*}\right)
\end{aligned}
$$

Since these four equations are highly non-linear, it is not possible to find an analytical solution to the system. However, numerical solutions may be obtained for specific parameters as we illustrate in the next section. Upon solving for the optimal investment and waiting regions, the capacity size is then scaled accordingly.

\section{$6 \quad$ Numerical Examples}

We use the following parameter values for our numerical examples:

- $r=0.10$

- $\mu=0.01$ 
- $P_{0}=25(\$ / \mathrm{MWh})$

- $c_{N}=20(\$ / \mathrm{MWh})$

- $c_{G}=40(\$ / \mathrm{MWh})$

- $T=2$ (years)

- $L=40$ (years)

- $\epsilon=2$

- $\delta_{N}=4.17 \times 10^{-5}\left(\$ / \mathrm{MWh}^{2}\right)$

- $\delta_{G}=2.09 \times 10^{-5}\left(\$ / \mathrm{MWh}^{2}\right)$

- $Q=8760 \times 500=4380000(\mathrm{MWh})$

The $\delta_{N}$ and $\delta_{G}$ parameters are calibrated so that a nuclear plant of $500 \mathrm{MW}$ capacity has an investment cost of $\$ 800$ million based on a reported cost of $\$ 1500 / \mathrm{kW}$ in [9]. A gas-fired power plant of the same capacity is assumed to be half as expensive to build. In addition, we assume a base value of $\sigma=0.20$, but allow it to vary in order to perform sensitivity analyses.

When a mutually exclusive investment opportunity in the two projects is considered, the value of the option to invest is dichotomous around the indifference price, $\tilde{P}=78.60$, at a relatively low level of uncertainty. In this example, it is the case that $F_{N}\left(P_{0}\right)>F_{G}\left(P_{0}\right)$ for $\sigma<0.152$, which leads to the situation in Fig. 1 . For an initial electricity price of $\$ 25 / \mathrm{MWh}$, this implies that it is optimal to wait until the electricity price hits a level of $P_{N}^{*}=52.13$ before investing in a nuclear power plant of capacity $424 \mathrm{MW}$ (as determined via Eq. (13)). In fact, for electricity prices between $\$ 52.13 / \mathrm{MWh}$ and $\$ 56.80 / \mathrm{MWh}$, it is optimal to invest immediately in a nuclear power plant with capacity that is scaled to maximize the NPV (as determined

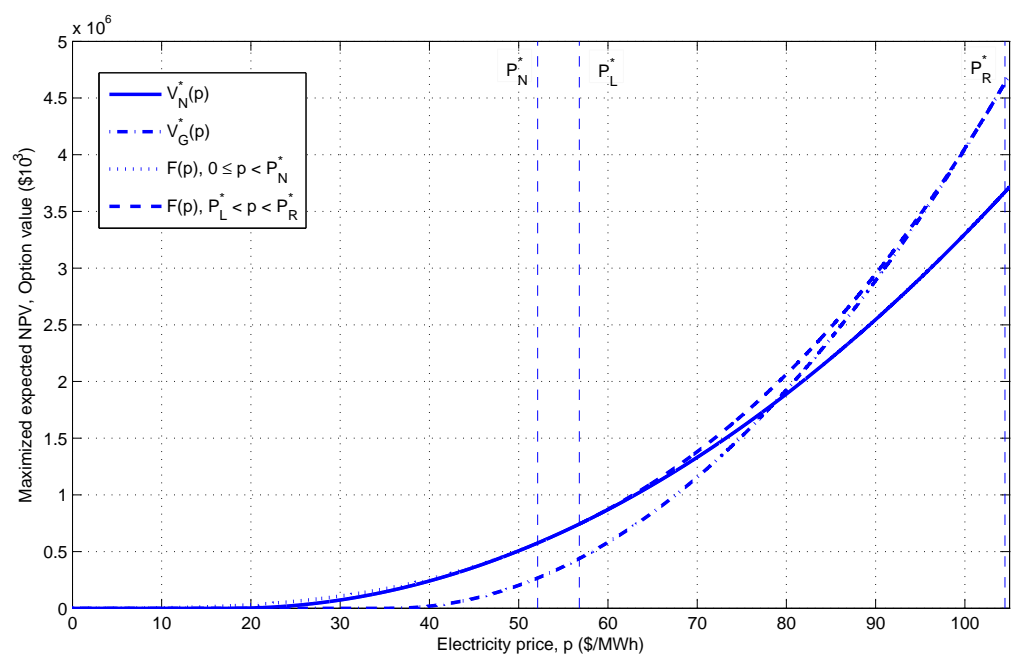

Figure 1: Maximized expected NPV and option value of a mutually exclusive investment opportunity in nuclear and gas power plants with optimal capacities $(\sigma=0.15)$ 


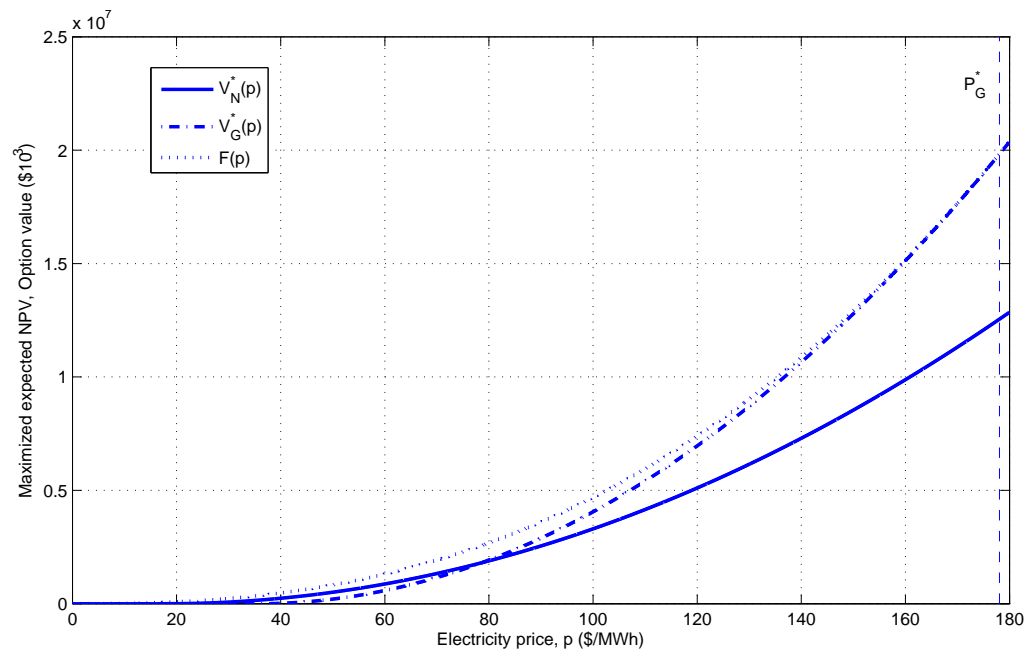

Figure 2: Maximized expected NPV and option value of a mutually exclusive investment opportunity in nuclear and gas power plants with optimal capacities $(\sigma=0.20)$

via Eq. (9)), i.e., it is optimal to construct an even larger plant. However, for an initial price in the range $P_{L}^{*}=\$ 56.80 / \mathrm{MWh}$ to $P_{R}^{*}=\$ 104.49$, it is optimal to wait: if the electricity price drops (rises) to the lower (upper) threshold, then it is optimal to invest immediately in a nuclear (gas) power plant. In the latter case, a gas-fired power plant of capacity $1702 \mathrm{MW}$ is constructed, where the optimal size is determined via Eq. (9). Finally, it should be noted that $P_{R}^{*}>P_{G}^{*}=\$ 104.27$, i.e., it is optimal to delay investment in the gas-fired power plant longer when the mutually exclusive option to proceed with nuclear is also considered. Intuitively, the inclusion of a second possible project increases the value of the entire investment opportunity, but it also makes investment in any specific technology less likely. For example, $F(\tilde{P})=\$ 1.957$ billion, which is greater than $F_{G}(\tilde{P})=\$ 1.952$ billion by $\$ 4.88$ million, i.e., a difference of $0.25 \%$.

As uncertainty increases, however, the immediate investment region in the nuclear technology shrinks until for $\sigma>0.152$ it disappears completely. In fact, for volatility estimates greater than 0.152 , the value of the option to invest in the gas technology dominates the one for the nuclear technology. Thus, it is preferable to skip the nuclear technology and consider only the option to invest in the gas one. Fig. 2 illustrates the maximized expected NPV and option value curves for $\sigma=0.20$. Since $F_{N}\left(P_{0}\right)=\$ 104$ million and $F_{G}\left(P_{0}\right)=\$ 146$ million, the value of the mutually exclusive option to invest is simply the value of the option to invest in the gas technology, i.e., $F(p)=F_{G}(p)$. As in the model for analyzing the investment timing and the plant sizing, we wait until the electricity price is $\$ 178.07 / \mathrm{MWh}$ before investing in a gas-fired power plant of capacity $3520 \mathrm{MW}$. Of course, if the initial price were higher than this threshold, $P_{G}^{*}$, then we would invest immediately in a larger power plant, which would be scaled using Eq. (9). Finally, Fig. 3 traces the effect of varying the volatility parameter on investment threshold prices and technology choices. The dichotomous waiting region appears only for low levels of uncertainty. 


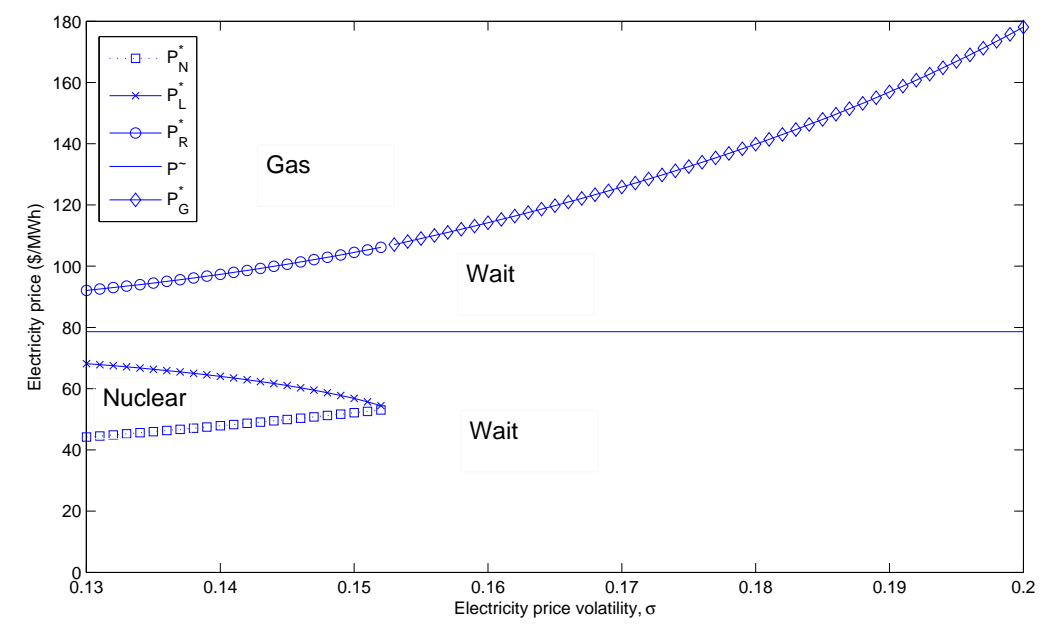

Figure 3: Investment threshold prices as a function of volatility with endogenous capacity sizing and mutually exclusive investment

\section{Conclusions}

Due to the ongoing deregulation of the electricity industry, investors in generation and transmission assets have more flexibility over their decisions. Such flexibility can encompass timing, sizing, and technology choice, to name a few. Under the regulated paradigm, relatively stable energy prices made such flexibility nearly obsolete as most investment opportunities could be appraised via the standard NPV approach. Uncertain prices, however, provide an incentive to defer decisions in order to receive more information especially when there is additional flexibility in the form of capacity sizing and technology choice.

In this paper, we have developed an analytical model to value to option to invest in mutually exclusive generation technologies when there exists flexibility over timing and sizing. We note first that due to the value of waiting, investment using the real options approach occurs later than via the now-or-never NPV one. Second, uncertainty affects not only the timing of investment, but also its scale, i.e., investors try to maximize their expected profit by waiting longer and building larger plants. Finally, consideration of mutually exclusive projects increases the option value of the entire investment opportunity while deferring adoption of any particular technology. Indeed, in a situation with a relatively low level of uncertainty, the investor may not be able to rule out the technology that performs better under relatively low long-term electricity prices, e.g., nuclear in our case, and, thus, has the additional incentive to wait longer.

\section{References}

[1] Wilson RB (1998) Architecture of power markets. Econometrica 70:1299-1340

[2] Hyman LS (2009) Restructuring electricity policy and financial models. Energy Economics, forthcoming 
[3] Dixit AK, Pindyck RS (1994) Investment under uncertainty. Princeton University Press, Princeton, NJ, USA

[4] McDonald R, Siegel D (1986) The value of waiting to invest. Quarterly Journal of Economics 101:707-727

[5] Ekern S (1988) An option pricing approach to evaluating petroleum projects. Energy Economics 10:91-99

[6] Deng S-J, Johnson B, Sogomonian A (2001) Exotic electricity options and the valuation of electricity and generation assets. Decision Support Systems 30:383-392

[7] Tseng C-L, Barz G (2002) Short-term generation asset valuation: a real options approach. Operations Research 50:297-310

[8] Deng S-J, Oren SS (2003) Incorporating operational characteristics and startup costs in option-based valuation of power generation capacity. Probability in the Engineering and Informational Sciences 17:155-181

[9] Rothwell G (2006) A real options approach to evaluating new nuclear power plants. The Energy Journal 27:37-53

[10] Gollier C, Proult D, Thais F, Walgenwitz G (2005) Choice of nuclear power investments under price uncertainty: Valuing modularity. Energy Economics 27:667-685

[11] Näsäkkälä E, Fleten S-E (2005) Flexibility and technology choice in gas fired power plant investments. Review of Financial Economics 14:371-393

[12] Siddiqui AS, Maribu K (2009) Investment and upgrade in distributed generation under uncertainty. Energy Economics 31:25-37

[13] Bøckman T, Fleten S-E, Juliussen E, Langhammer H, Revdal J (2008) Investment timing and optimal capacity choice for small hydropower projects. European Journal of Operational Research 190:255-267

[14] Takashima R, Naito Y, Kimura H, Madarame H (2007) Decommissioning and equipment replacement of nuclear power plants under uncertainty. Journal of Nuclear Science and Technology 44:1347-1355

[15] Décamps J-P, Mariotti T, Villeneuve S (2006) Irreversible investment in alternative projects. Economic Theory 28:425-448

[16] Huisman KJM, Kort PM (2003) Strategic investment in technological innovations. European Journal of Operational Research 144:209-233

[17] Fleten S-E, Maribu KM, Wangensteen I (2007) Optimal investment strategies in decentralized renewable power generation under uncertainty. Energy 32:803-815

[18] Wickart M, Madlener R (2007) Optimal technology choice and investment timing: A stochastic model of industrial cogeneration vs. heat-only production. Energy Economics 29:934-952 
[19] Takashima R, Goto M, Kimura H, Madarame H (2008) Entry into the electricity market: uncertainty, competition, and mothballing options. Energy Economics 30:1809-1830

[20] Dangl T (1999) Investment and capacity choice under uncertain demand. European Journal of Operational Research 117:415-428

[21] Bar-Ilan A, Strange WC (1996) Investment lags. American Economic Review 86(3):610-622 